\title{
Radial Distribution of Dilated Intercellular Spaces of the Esophageal Squamous Epithelium in Patients with Reflux Disease Exhibiting Discrete Endoscopic Lesions
}

\author{
M. Vieth ${ }^{a}$ R. Fiocca ${ }^{b}$ J. Haringsma ${ }^{c}$ J. Delarived P.H. Wieseld W. Tame \\ G.N.J. Tytgat ${ }^{f}$ J. Dent ${ }^{\mathrm{e}}$ A. Edebo ${ }^{g} \quad$ L. Lundellg $\quad$ M. Stolte ${ }^{\text {h }}$ \\ a Institute of Pathology, Otto-von-Guericke University Magdeburg, Magdeburg, Germany; b Institute of Pathology, \\ University of Genova, Genova, Italy; ' Department of Gastroenterology, Erasmus MC-University Medical Center, \\ Rotterdam, The Netherlands; dDepartment of Gastroenterology, Centre Hospitalier Universitaire Vaudois, \\ Lausanne, Switzerland; eDepartment of Gastrointestinal Medicine, Royal Adelaide Hospital, North Terrace, \\ Adelaide, Australia; fDepartment of Gastroenterology, Academic Medical Center, University of Amsterdam, \\ Amsterdam, The Netherlands; 9Department of Surgery, Sahlgrenska University Hospital, Göteborg, Sweden; \\ hInstitute of Pathology, Bayreuth, Germany
}

\section{Key Words}

Gastro-esophageal reflux disease - Dilatation of intercellular spaces · Histology · Interobserver variation • Squamous epithelium

\begin{abstract}
Introduction: Dilatation of intercellular spaces of the esophageal squamous epithelium has been suggested as a marker of early acid reflux-induced damage. This change is a potentially useful addition to histomorphological changes that represent so called minimal endoscopic lesions. We have assessed dilatation of intercellular spaces with regard to: (1) interobserver variability, and (2) whether the incidence of this varies between 'red streaks' and the adjacent normal looking squamous epithelium. Methods: Esophageal biopsies from 44 patients with chronic gastro-esophageal reflux (GERD) were evaluated. At endoscopy, these patients had one or more red streaks on the tops of the mucosal folds in the distal esophagus. Biopsies were taken from the red streaks and from the normal-appearing mucosa $1 \mathrm{~cm}$ lateral to the
\end{abstract}

red streaks. Biopsies were assessed in a blinded fashion by two independent pathologists (MV \& RF). Criteria for assessing intercellular space dilatation were evaluated and agreed on prior to the study. Results: Good interobserver agreement was recorded $(\mathrm{kappa}=0.82$ at the streaks and 0.77 for the control tissues) for absence/presence of intercellular space dilatation. Red streak and control biopsies differed significantly ( $p=0.0001$ ), with respect to presence of dilated intercellular spaces, with $90.5 \%$ of the former demonstrating this as present compared to $56.1 \%$ in the controls. Conclusion: This study supports the concept that esophageal mucosal minimal changes due to reflux is localised and that dilatation of intercellular spaces is an early sign of reflux-induced epithelial damage. The low interobserver variability in the assessment of intercellular space dilatation suggests that this may be a useful variable for assessment of early signs of acid-reflux induced damage to the squamous epithelium of the esophagus by use of light microscopy. Copyright $\odot 2004$ S. Karger AG, Basel

\begin{tabular}{ll}
\hline KARGER & ( ) 2004 S. Karger AG, Basel \\
0257-2753/04/0222-0208\$21.00/0 \\
$\begin{array}{l}\text { Fax +4161306 12 34 } \\
\text { www.karger.com }\end{array}$ & $\begin{array}{l}\text { Accessible online at: } \\
\text { www.karger.com/ddi }\end{array}$
\end{tabular}

PD Dr. med. M. Vieth

Institute of Pathology, Otto-von-Guericke University Magdeburg, Leipzigerstrasse 44 DE-39120 Magdeburg (Germany)

Tel. +49 9214005602 , Fax +49 9214005609

E-Mail Pathologie.Klinikum-Bayreuth@t-online.de 


\section{Introduction}

The Los Angeles classification for the scoring of the severity of reflux esophagus has been gained increasing recognition from both the scientific and clinical perspectives $[1,13]$. This classification evaluates the extent of endoscopically visible mucosal breaks and requires that visible mucosal breaks be present for the endoscopic diagnosis of reflux oesophagitis. The Los Angeles Classification group has rejected the use of so-called minimal changes to improve the sensitivity of the endoscopic diagnosis of reflux disease. This decision was taken because it has previously been shown that minor distal esophageal mucosal changes $[3,6,18]$ traditionally thought to be diagnostic of reflux disease cannot be reliably recognized by endoscopists $[8,9,11]$. Such changes that have been evaluated include erythema, friability, mucosal oedema and blurring of the squamocolumnar junction. Lack of validated 'minimal change' endoscopic criteria is a significant limitation of the utility of endoscopy for diagnosis of reflux disease, since the majority of sufferers from reflux disease have no mucosal breaks [7].

Various histological changes of the epithelium of the distal esophagus have been proposed as useful for the diagnosis of reflux disease in the absence of endoscopic mucosal breaks. These include basal cell hyperplasia, elongation of the papillae and intraepithelial infiltration of inflammatory cells, all of which can be assessed with light microscopy [2, 10]. Recently [17], intercellular space dilatation (ISD) of more than $2.4 \mu \mathrm{m}$ between esophageal squamous mucosal cells in at least one site [16] has been reported to be present in endoscopy negative GERD patients. Quite extensive experimental studies have elucidated the potential mechanisms involved in the pathogenesis of ISD $[15,12]$.

In a previous analysis, our group showed that the histomorphological correlate of so-called red streaks in the distal esophagus are streaks of newly re-epithelized squamous epithelium with underlying capillary-rich granulation tissue [18]. In this study, we showed that basal cell hyperplasia and elongation of papillae are useful for distinguishing biopsies taken directly from red streaks from adjacent normal-appearing mucosa. The aims of the present study were, firstly, to assess the accuracy by which trained pathologists can assess ISD and secondly whether ISD in endoscopically defined esophageal 'red streaks' indicative of reflux esophagitis varies from the adjacent normally looking squamous epithelium.
Table 1. Demographic data for 44 patients with one or more longitudinal red streaks visible just above the z-line in the distal esophagus

\begin{tabular}{lc}
\hline Number of patients & 44 \\
Male:female ratio & $1.5: 1(29: 15)$ \\
Mean age (years \pm SD) & $51.3 \pm 16.2$ \\
Patients with one red streak & $34(77 \%)$ \\
Patients with more than one red streak & $10(23 \%)$ \\
\hline
\end{tabular}

\section{Patients and Methods}

The general methodology has been presented previously [18] and so is summarized briefly here.

\section{Patients}

44 patients (table 1) with long-standing heartburn and/or acid regurgitation were included in a multicenter prospective study. The major inclusion criterion was the presence of one or more red streaks in the distal esophagus at the time of routine diagnostic endoscopy. Patients with white exudate or slough covering any part of the red streaks were not included. All patients had been referred to the outpatient department of 4 European and one Australian University Hospital for upper gastrointestinal endoscopy. Forty-two of these patients were described in detail in a prior study [18] with regard to a histomorphological correlate of so-called red streaks in the distal esophagus. The present study is a separate analysis of the same patients now with regard to the finding of ISD.

\section{Endoscopy}

Biopsies were obtained from each red streak. An additional biopsy was thereafter taken from the normal-appearing mucosa $1 \mathrm{~cm}$ lateral to the red streak. The latter biopsies are subsequently referred to as 'control' biopsies. The exact endoscopic protocol has already been described previously [18].

\section{Histology}

The biopsy specimens were fixed in $10 \%$ buffered formalin then dehydrated in an increasing alcohol concentration followed by xylol, oriented vertically and embedded in paraffin. The 4- $\mu$ m-thick stepped sections were deparaffinized and stained with HE. All slides were assessed independently by two pathologists (M.V. and R.F.) who interpreted the biopsies without knowledge of their location. Intercellular dilation was assessed by light microscopy as present or absent without any further grading whenever the intercellular space was more than $2.4 \mu \mathrm{m}$ [16], provided this was present diffusely ( $>25 \%$ of the specimen) in the parabasal layer of the squamous epithelium.

\section{Statistics}

Statistical analysis was performed using SPSS Software (SPPS, Chicago, Ill., USA) to assess differences in presence or absence of intercellular spaces between the two biopsy locations (red streak versus control biopsies). Paired comparisons of red streak and control biopsies taken at one level within one patient, were performed with the non-parametric Pearson $\chi^{2}$ test. A probability ( $\mathrm{p}$ value) of below 0.05 was considered to be statistically significant. Kappa values were calculated when assessing the interobserver variation [4]. Kappa was 


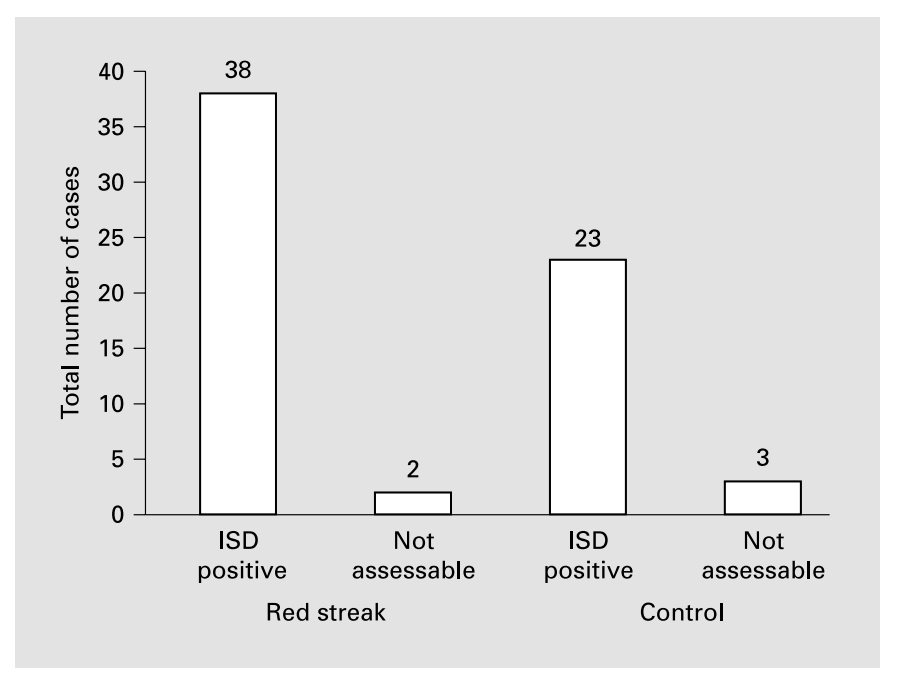

Fig. 1. Presence of dilated intercellular spaces in biopsies obtained from red streaks compared to the control biopsies.

determined through $\mathrm{K}=\left(\mathrm{CR}_{\mathrm{b}}-\mathrm{CR}_{\mathrm{e}}\right):\left(1-\mathrm{CR}_{\mathrm{e}}\right)$. In this equation $\mathrm{CR}_{\mathrm{b}}$ describes the concordance whereas $\mathrm{CR}_{\mathrm{e}}$ represents the expected rate of concordance [4]. A kappa value of $0.21-0.40$ is considered as fair, $0.41-0.60$ as moderate, $0.61-0.80$ as a substantial and $0.81-1.00$ as an almost perfect concordance [5].

\section{Results}

\section{Interobserver Variability}

Biopsies were read independently for ISD on two occasions by two investigators ( $\mathrm{MV}$ and $\mathrm{RF}$ ), once before these pathologists had met prior to a formal definition of criteria for ISD and again after this.

\section{First Assessment prior to Agreement on Criteria for \\ ISD}

Thirty-three of 42 biopsies from the red streaks (excluding 2 cases in whom biopsies were too small and/or too tangential) were concordantly diagnosed as positive for ISD and 4 were concordantly diagnosed as negative, while in 5 of the biopsies from red streaks there was discordance as to the presence of ISD, 4 cases being diagnosed negative by MV and 1 case negative by RF. For the control biopsies from 41 patients ( 3 were excluded because biopsies were either too small or too tangential). 15 biopsies were concordantly diagnosed as negative for ISD and 15 concordantly positive, while 11 cases proved discordant, 10 cases being diagnosed negative by MV and 1

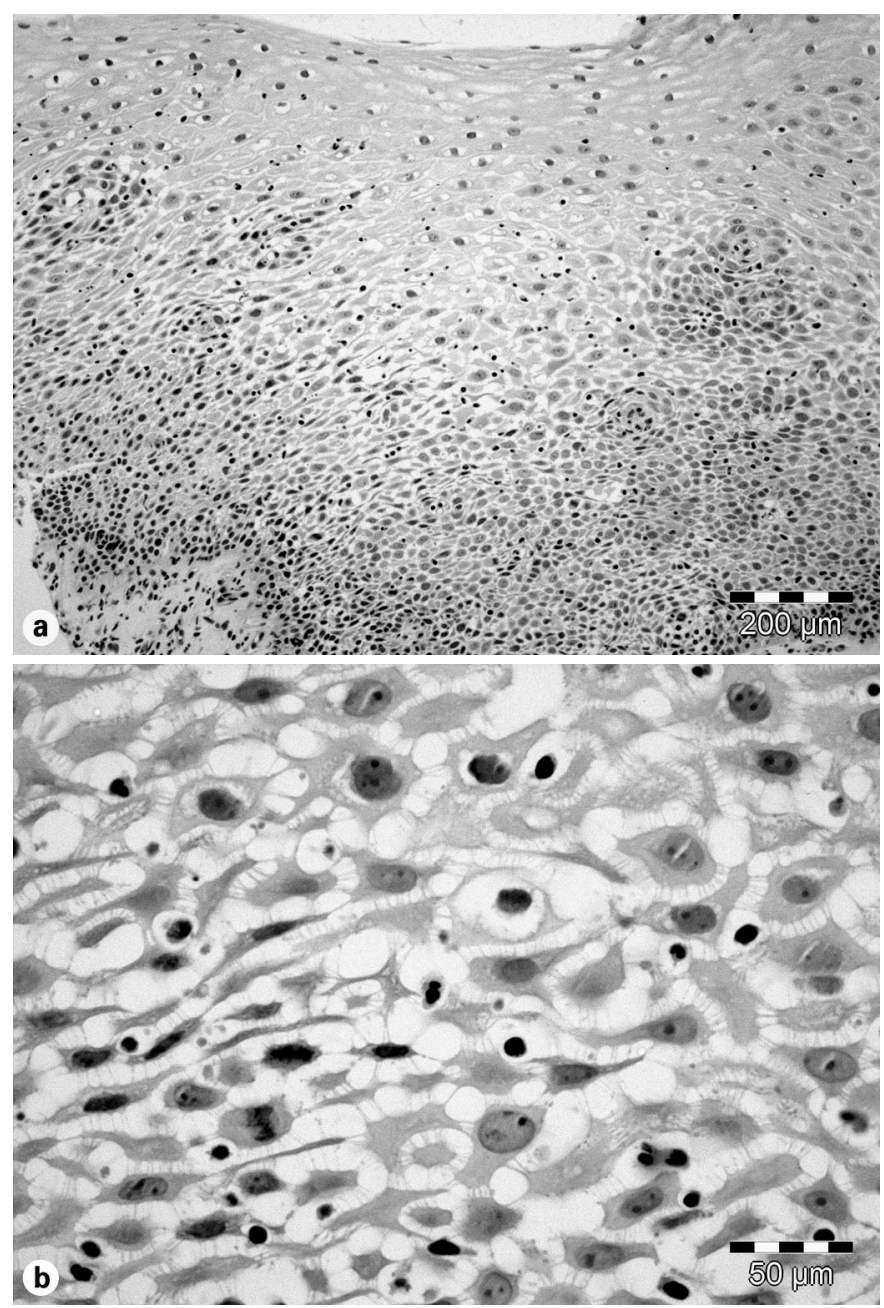

Fig. 2. Irregular dilation of intercellular space with cytoplasmatic invaginations and vacuole formation in the basal and deep prickle layers of a biopsy obtained from a red streak in reflux disease. a HE. $\times 100$. b HE. $\times 400$.

further case was excluded by RF due to a too tangential biopsy (kappa $=0.6$ for red streak and 0.53 for the control biopsies).

\section{Second Assessment after Agreement on Criteria for ISD}

On the second reading of the biopsies the following results were obtained, 38 of 42 biopsies from the red streaks were concordantly diagnosed positive and 4 concordantly negative, while 1 of the biopsies from the red streak was still diagnosed as negative by RF but positive by MV. The control biopsies were concordantly diagnosed negative in 16 cases and positive in 23 cases. One 
case was diagnosed discordantly, being scored as positive by RF. A further case was judged by RF as not eligible for evaluation (kappa $=0.82$ for the red streak and 0.77 for the control biopsies).

Biopsies from red streaks contained ISD significantly $(p=0.0001)$ more frequently than the control biopsies. ISD was seen in $90.5 \%$ (38 of 42) of the biopsies from the red streak. By comparison only $56.1 \%$ (23 of 41 ) of the control biopsies showed dilated intercellular spaces. Twenty-two out of 42 cases (52.4\%) showed intercellular dilation in both the biopsy specimens from red streaks as well as the control biopsies (fig. 1,2).

\section{Discussion}

We recently reported that endoscopically recognized red streaks of the distal esophageal epithelium of GERD patients displayed consistent histomorphological findings in the form of basal cell layer hyperplasia and elongation of the papillae and underlying granulation tissue rich in capillaries [18] which contrasted to the situation found in the adjacent normal looking squamous epithelium. These observations validated the endoscopic differentiation of mucosal breaks used in the Los Angeles system for grading of reflux esophagitis which was originally based on the premise that acid-peptic injury of the esophageal epithelium expands radially from the top of the mucosal folds $[1,13]$. Studies by Orlando and co-workers $[15,17]$ have shown that expansion of the intercellular spaces, assessed by electron microscopy, is an early and consistent finding in the process of acid-peptic injury to squamous epithelium.

Solcia et al. [16] have reported that ISD can be assessed in biopsies by light microscopy, thus making assessment of this variable far more practical. In asmyptomatic controls ISD are found only in 8\% [16]. Our data from GERD patients confirm that ISD can be assessed by light microscopy with excellent agreement between pathologists, but show the need for prior training on the recognition of this change. The interobserver variation in the initial assessment of ISD prior to consensus on criterion was mainly due to difficulties in scoring less marked intercellular space dilatation. After mutual agreement on the criteria for the assessment we achieved kappa values around 0.8, suggesting that ISD can be readily and reliably incorporated as a routine part of the light microscopy evaluation of the distal squamous epithelium in reflux disease.

In our previous histological study on the morphological counterparts to the red streaks confined to the mucosal folds in reflux disease, we found evidence to support the notion that red streaks arise from acid-peptic damage. This damage is evident as elongation of papillae, and hyperplasia of the basal cell layer underlying highly vascularized granulation tissue, often in combination with a thin layer of regenerating squamous epithelium. These changes reflect reactive-regenerative processes [18]. Our data on ISD add support to the hypothesis that there are loci minoris resistencae in the distal esophagus since the lesions were so circumscribed. Especially the tops of the mucosal fold should be regarded as primary target for mucosal damage in reflux disease. It has not been the routine to target the tops of esophageal mucosal folds when biopsies have been used to assess presence of reflux disease. Until proven otherwise, it seems appropriate that biopsies taken for this purpose should be so targeted, as otherwise, important information could be missed.

Results from our present and previously published study support the concept of focal nature of refluxinduced injuries to the esophageal epithelium [18]. It is therefore to be expected that at least some of the biopsies would show ISD, if this is a useful marker of excessive acid-peptic exposure.

In conclusion, our data add to existing data which indicate that ISD of the squamous epithelium is a marker for GERD, since it is closely connected with endoscopically recognizable mucosal red streaks confined to mucosal areas exposed to acid peptic damage. Studies are in progress in patients and healthy controls to evaluate the sensitivity and specificity of ISD and how this might change in response to highly effective acid inhibition. 


\section{References}

1 Armstrong D, Monnier P, Nicolet M, Blum AL, Savary M: Endoscopic assessment of esophagitis. Gullet 1991;1:63-67.

2 Brown LF, Goldman H, Antonioli DA: Intraepithelial eosinophils in endoscopic biopsies of adults with reflux esophagitis. Am J Surg Pathol 1984;8:899-905.

3 Bytzer P, Havelund T, Møller Hansen J: Interobserver variation in the endoscopic diagnosis of reflux esophagitis. Scand J Gastroenterol 1993;28:119-125.

4 Goodman Leo A, Kruskal William H: In: Measures of Association for Cross Classifications. New York, Springer, 1997:1-45.

5 Haas M: Statistical methodology for reliability studies. J Manipulative Physiol Ther 1991;14: 119-132.

6 Hatlebakk JG, Berstad A: Endoscopic grading of reflux esophagitis: What observations correlate with gastro-esophageal reflux? Scand J Gastroenterol 1997;32:760-765.

7 Hollenz M, Stolte M, Labenz J: Prevalence of gastro-esophageal reflux disease in genera practice. Dtsch Med Wochenschr 2002;127: 1007-1012.
8 Ismail-Beigi F, Horton PF, Pope CE II: Histological consequences of gastresophageal reflux in the distal esophagus in man. Gastroenterology 1970;58:163-174.

9 Ismail-Beigi F, Pope CE II: Distribution of the histological changes of gastroesophageal reflux in the distal esophagus of man. Gastroenterology 1974;66:1109-1113.

10 Jarvis LR, Dent J, Withehead R: Morphometric assessment of reflux esophagitis in fibreoptic biopsy specimens. J Clin Pathol 1985;38: 44-48.

11 Kobayshi S, Kasugai T: Endoscopic and biopsy criteria for the diagnosis of esophagitis with fiberoptic esophagoscope. Am J Dig Dis 1974; 19:345-352.

12 Long JD, Orlando RC: Esophageal submucosal glands: structure and function. Am J Gastroenterol 1999;94:2818-2124.

13 Lundell LR, Dent J, Bennett JR, Blum AL, Armstrong D, Galmiche JP, Johnson F, Hongo M, Richter JE, Spechler SJ, Tytgat GN, Wallin L: Endoscopic assessment of esophagitis - clinical and functional correlates and future validation of the Los Angeles classification. Gut 1999:45:172-180.
14 Murphy DW, Yuan Y, Castell DO: Does the intraesophageal $\mathrm{pH}$ probe accurately detect acid reflux? Dig Dis Sci 1989;34:649-656.

15 Orlando RC: Why is the high grade inhibition of gastric acid secretion afforded by proton pump inhibitors often required for healing of reflux esophagitis? An epithelial perspective. Am J Gastroenterol 1996:91:1692-1696.

16 Solcia E, Villani L, Luinetti O, Trespi E, Strada E, Tinelli C, Fiocca R: Altered intercellular glycoconjugates and dilated intercellular spaces of esophageal epithelium in reflux disease. Virchows Arch 2000;436:207-216.

17 Tobey NA, Carson JL, Alkiek RA, Orlando RC: Dilated intercellular spaces: A morphological feature of acid reflux damaged human esophageal epithelium. Gastroenterology 1996; 111:1200-1205.

18 Vieth M, Haringsma J, Delarive J, Wiesel PH, Tam W, Dent J, Tytgat GN, Stolte M, Lundell $\mathrm{L}$ : Red streaks in the esophagus in patients with reflux disease: Is there a histomorphological correlate? Scand J Gastroenterol 2001;36: 1123-1127. 